\title{
Side-pumped neodymium laser with self-adaptive, nonreciprocal cavity
}

\author{
M. KASKOW*, L. GALECKI, W. ZENDZIAN, L. GORAJEK, and J.K. JABCZYNSKI \\ Institute of Optoelectronics, Military University of Technology, ul. Gen. Sylwestra Kaliskiego 2, \\ 00-908 Warsaw, Poland
}

We demonstrate the generation of a near-diffraction-limited beam in a diode-side-pumped Nd:YAG slab laser with a nonreciprocal, self-adaptive cavity. The energy output of $228 \mathrm{~mJ}$ with $17.2 \%$ slope efficiency and $17.3 \%$ optical-to-optical efficiency was obtained.

Keywords: side-pumped, neodymium, solid-state, garnet, laser, four-wave mixing.

\section{Introduction}

The near infrared laser sources are very attractive devices which are widely used in the industry, healthcare, environmental monitoring and military. However, certain particular applications like, i.e., remote sensing or target designation require high-energy output of order of few hundreds of $\mathrm{mJs}$ with diffraction-limited beam quality. In order to meet those conditions, a special approach in the laser construction must be done. The progress done in recent years in 2D laser diode arrays development enabled to build compact side-pumping modules which can provide tens of kWs of peak power confined to a few square millimetres area. On the other hand, intensive pumping and heat dissipation induce strong thermal effects which favour generation in higher transverse modes. Additionally, it significantly degrades the spatial quality and decreases the overall performance of the laser system. There are several techniques, i.e., injection-seeding $[1,2]$, grazing-incidence-angle $[3,4]$ or self-adaptive resonators based on phase conjugation via gain saturation mechanism [5-15], that allow to generate near-diffraction-limited beam output in the presence of a high inhomogeneity of the gain spatial profile. In this paper we demonstrate the results of a self-adaptive Nd:YAG laser with intra-cavity amplifier with an open-loop, nonreciprocal cavity containing phase conjugate mirror.

\section{Principle of operation}

The self-adaptive resonator scheme is depicted in Fig. 1. Initially it is an open-loop cavity without any feedback. The intra-cavity waves develop from the spontaneous emission. Due to the gain saturation mechanism in the main head, waves $E_{1}$ and $E_{3}$ interfere constructively in the gain medium

\footnotetext{
*e-mail: mateusz.kaskow@wat.edu.pl
}

(intersecting under $\theta$ angle which determines the gain-fringes period) forming a transmissive gain grating, whereas $E_{2}$ and $E_{3}$ form a reflective gain grating. The phase conjugate $E_{4}$ wave [see Eq. (1)] is generated as a consequence of the diffraction of the $E_{1}$ and $E_{2}$ on reflective and transmissive gratings, respectively

$$
E_{4} \infty E_{1} E_{2} E_{3}^{*}
$$

$E_{4}$ propagates in the resonator and converts to $E_{2}$ and $E_{1}$. As soon as the gain-hologram is written in the active medium and the threshold condition is fulfilled, this process builds up to a point, when the phase conjugate mirror (PCM) is formed as a result of nondegenerate four-wave mixing mechanism inside an active medium. The two polarizers, a half wave plate and a Faraday rotator create a nonreciprocal transmission element (NRTE) which allows to regulate the amplitude of counter-propagating waves, thus to control of the diffraction efficiency of the gain gratings and to prevent the saturation of the amplifier by a counter-propagating wave. The PCM provides feedback, closes

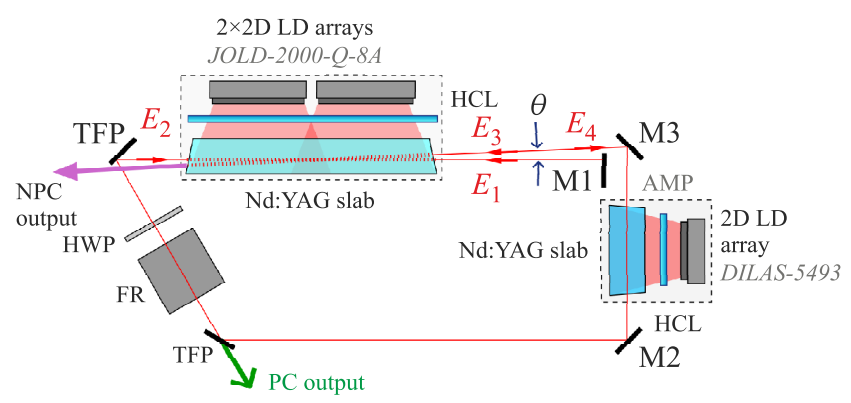

Fig. 1. Scheme of the self-adaptive nonreciprocal cavity: TFP thin-film polarizer; $\mathrm{HCL}$ - cylindrical lens; HWP - half wave plate; FR - Faraday rotator; $\theta$ - beam intersection angle; green arrow phase-conjugate output, purple arrow - non-phase conjugate output; AMP - amplifying stage. 
the loop and cancels the wavefront aberrations. Once the gain saturates, the diffraction efficiency of the gain grating significantly decreases and the output is generated as a series of a few hundred-ns-long pulses. In a certain, particular case, the gathered energy can be extracted in a single, few-tens to hundred nanoseconds pulse with near-diffraction-limited quality [10]. Hence, the gain gratings dynamics behave as a passive Q-switch. Furthermore, it acts as a spectral filter, as well. When certain conditions are met, only one longitudinal, fundamental mode is generated and laser operates in a single frequency regime. According to Ref. 9, in order to overcome the generation threshold, the small-signal gain has to be $g_{0} L \geq 8.5$. The main amplifier provided gain product of 7.2. We increased it by introduction of an additional amplifying stage (AMP), which will be described later.

\section{The Nd:YAG laser setup}

The $1 \%$ at. Nd:YAG single crystal with dimensions of $4 \times 4 \times 72 \mathrm{~mm}^{3}$ was applied as a gain medium in the laser oscillator head. Both $4 \times 4 \mathrm{~mm}^{2}$ facets were anti-parallel bevelled under $1^{\circ}$ angle in order to prevent the spontaneous emission amplification. The pumping module consisted of two 2D laser diode arrays (Jenoptik JOLD-2000-Q-8A) and a single cylindrical lens $(f=16 \mathrm{~mm})$. The emitters bars did not have fast-axis-collimation lenses. The emission wavelength stabilized at $20^{\circ} \mathrm{C}$ was measured to be $808 \mathrm{~nm}$ $(F W H M=3 \mathrm{~nm})$. For a pump duration $t_{p}=0.25 \mathrm{~ms}$ and repetition rate $P R F=2 \mathrm{~Hz}$, the maximum combined pump energy was slightly above $1 \mathrm{~J}$. The pump radiation was confined inside the gain medium to a $60 \mathrm{~mm}$-wide, $2.5 \mathrm{~mm}$ -high and 4 mm-deep excited area.

The amplifying stage consisted of the $1 \%$ at. Nd:YAG gain medium $\left(4 \times 4 \times 25 \mathrm{~mm}^{3}\right.$, facets bevelled under $\left.2^{\circ}\right)$ pumped with a single, fast-axis-collimated 2D laser diode array (DILAS-5493). The pump radiation was tightly focused by means of a single cylindrical lens $(f=50 \mathrm{~mm})$. Resulting excited volume in the active medium was $14.3 \times 0.25 \times 4 \mathrm{~mm}^{3}$. The emission wavelength stabilized at $25^{\circ} \mathrm{C}$ was measured to be $808 \mathrm{~nm}(F W H M=2.4 \mathrm{~nm})$. The pump source provided up to $0.5 \mathrm{~J}$ of energy in a $0.25 \mathrm{~ms}$ duration. Total resonator length was $L_{r e z}=3.3 \mathrm{~m}$. Due to a very low repetition rate of $2 \mathrm{~Hz}$, the influence of thermal effects was neglected.

\section{Experimental results}

\subsection{Diode-pumped Nd:YAG self-adaptive oscillator}

The experiments were carried out in a laser with the amplifying stage (AMP) turned off. The pump duration measured at full width at half maximum was $t_{p}=0.25 \mathrm{~ms}$ (FWHM). The energetic characteristics measured for different beam intersection angles $\theta$ is shown in Fig. 2.

This angle should be minimized, however, too low value, in the presence of high gain, will cause parasitic lasing

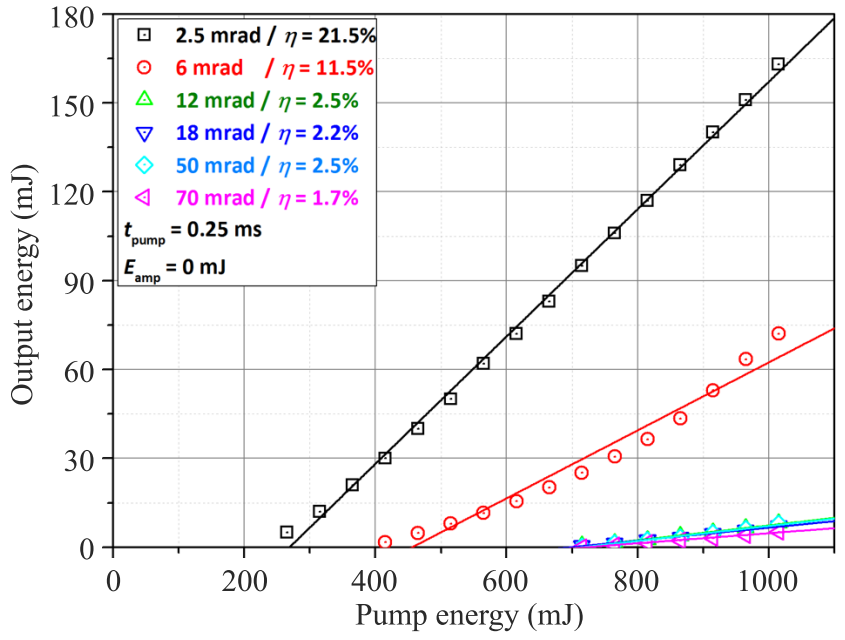

Fig. 2. Output energy $v s$. pump energy of laser oscillator for different beam intersection angles.

due to feedback provided by mirror M3. In our case, the best results were achieved for $\theta=2.5 \mathrm{mrad}$. The longest interference path contributes to decrease of the threshold and to increase of the gain grating diffraction efficiency. Output energy of $E_{\text {out }}=163 \mathrm{~mJ}$ with slope efficiency $\eta=21.5 \%$ and optical-to-optical efficiency $17.2 \%$ were achieved. For beam intersection angle above $12 \mathrm{mrad}$, slope efficiency was very low and remained below $3 \%$. The time trace of output pulse (registered with Thorlabs DET025AFC 2 GHz photodiode) is depicted in Fig. 3.

Figure 3(a) shows an oscillogram of output pulse burst. It can be explained as follows: high gain allows the gain grating to build up very fast, what in consequence increases its diffraction efficiency. The threshold is quickly overcome and the first pulse is generated. The upper laser level is depopulated and since there is enough gain provided by the pump, the scheme repeats itself and continues to generate other pulses in the series, until the pump is depleted. The generation of series of pulses was accompanied by a strong temporal and energetic jitter. Figure 3(b) depicts first pulse in the series. It was strongly modulated due to multimode generation. The contrast of the population grating was too low to provide desired mode selectivity. The pulse width measured at FWHM was of $746 \mathrm{~ns}$. The change of the retardation introduced by wave plate did not enforce single pulse generation. It decreased the energy output and increased the threshold. Reducing pump energy to $190 \mathrm{~mJ}$ allowed inefficient generation of a single pulse with energy of only $E_{p}=1.1 \mathrm{~mJ}$. The beam intensity spatial profile (Thorlabs BP-106 VIS CCD Beam Profiler) registered in far field is shown in Fig. 4.

The generated beam was astigmatic with an elliptical profile. The beam quality parameter $M^{2}$ was measured to be 2.6 and 2.5 whereas the full divergence angle was $1.4 \mathrm{mrad}$ and $1.9 \mathrm{mrad}$ in $\mathrm{X}$ and $\mathrm{Y}$ planes, respectively. The change of the beam intersection angle up to $40 \mathrm{mrad}$ did not significantly influence on the spatial quality. Above that value, the generation of higher transverse modes was obtained. 


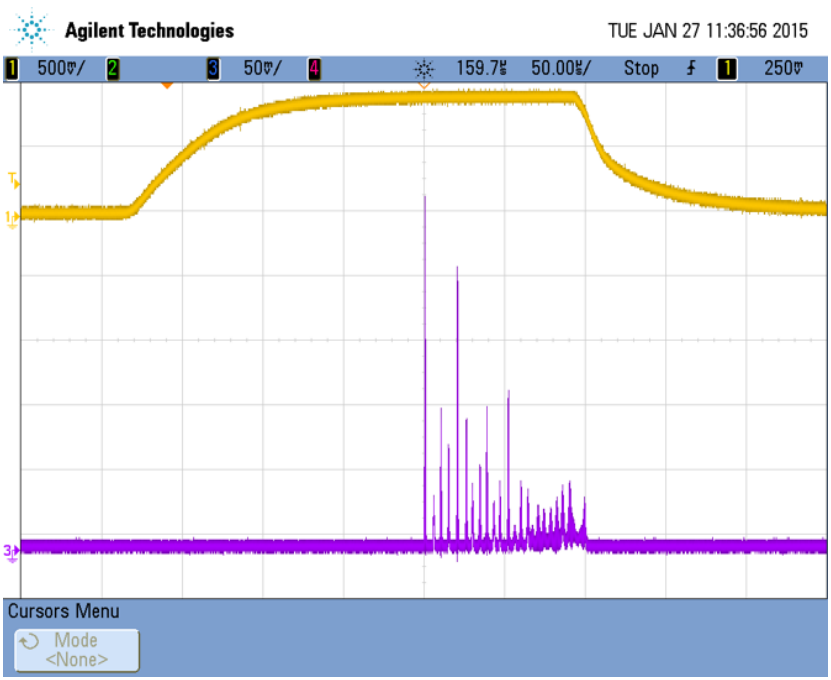

(a)

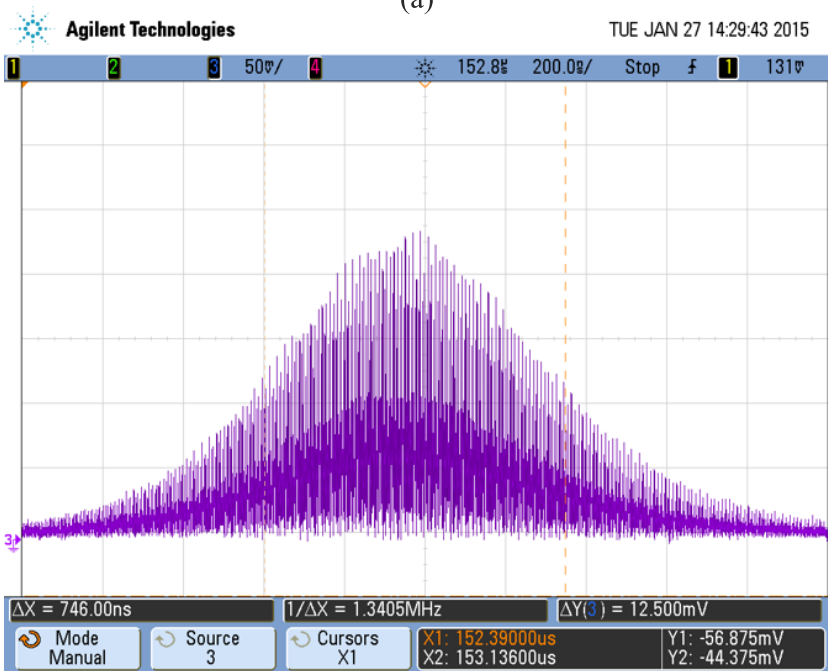

(b)

Fig. 3. Time trace of output pulse in relation to pump pulse (purple laser; yellow - pump).

\subsection{Diode-pumped Nd:YAG amplifier}

In the next step the amplifying stage was incorporated (see Fig. 1). The pump duration was set to $t_{a m p}=0.15 \mathrm{~ms}$. The delay between the amplifier and oscillator pump pulses was optimized to achieve highest pulse energy at the output. The pump energy of the oscillator remained at the maximum value. The output energy vs. total pump energy plots measured for different beam intersection angles are depicted in Fig. 5. Due to technical limitations of the laser diode driver we were unable to decrease the pump current below $100 \mathrm{~A}$, thus the minimal combined pumping energy was approx. $1.1 \mathrm{~J}$.

For beam intersecting at angle of $2.5 \mathrm{mrad}$, energy output of $228 \mathrm{~mJ}$ with a $17.2 \%$ slope efficiency and a $17.3 \%$ optical-to-optical efficiency was obtained. The output time trace consists of a pulse burst followed by a quasi-cw generation [see Fig. 6(a)].

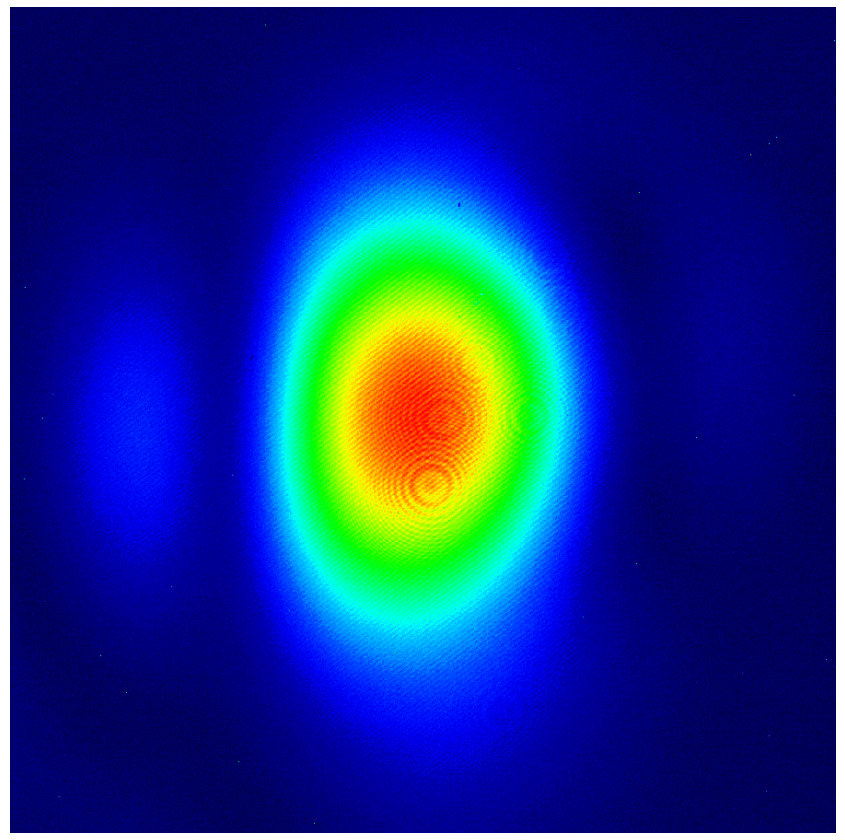

Fig. 4. Beam intensity spatial profile for $\theta=2.5 \mathrm{mrad}$.

Although the gain is higher (as well as the gain grating contrast), the grating dynamic and a low generation threshold did not allow to generate a single pulse. The effect saturates relatively fast and gain grating stops behaving as a Q-switch and favours q-cw generation. The first pulse in the series is less modulated [Fig. 6(b) vs. Fig. 3(b)] and its duration was measured to be 425 ns. The change of the wave plate retardation did not enforce single pulse generation. It influences only on the threshold and the efficiency. The beam intensity spatial profile (Thorlabs BP-106 VIS CCD Beam Profiler) registered in far field is shown in Fig. 7.

The beam quality parameter $M^{2}$ did not change significantly. It was measured to be 2.5 and 2.4 in X and Y planes, respectively. Beam divergence remained unchanged.

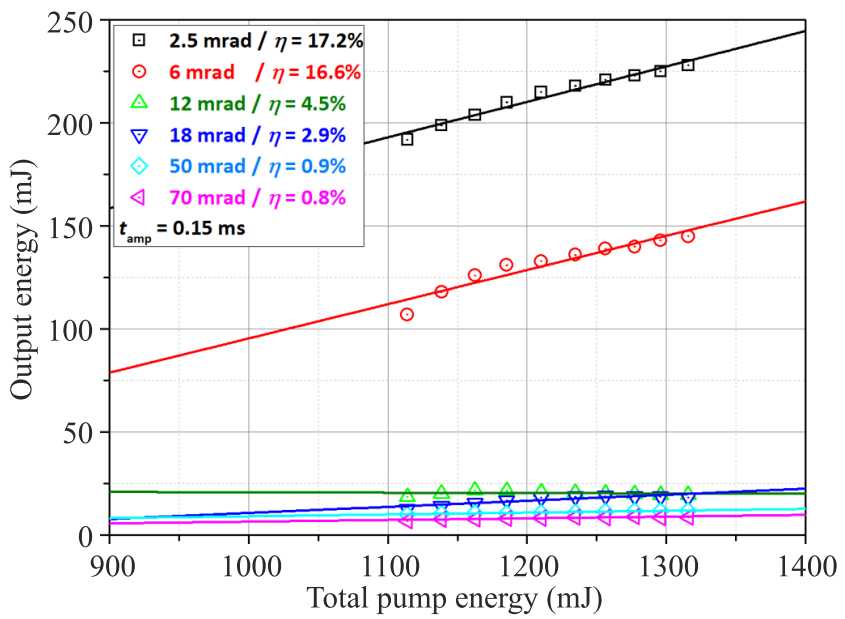

Fig. 5. Output energy $v s$. total pump energy of the laser with amplifier for different beam intersection angles. 


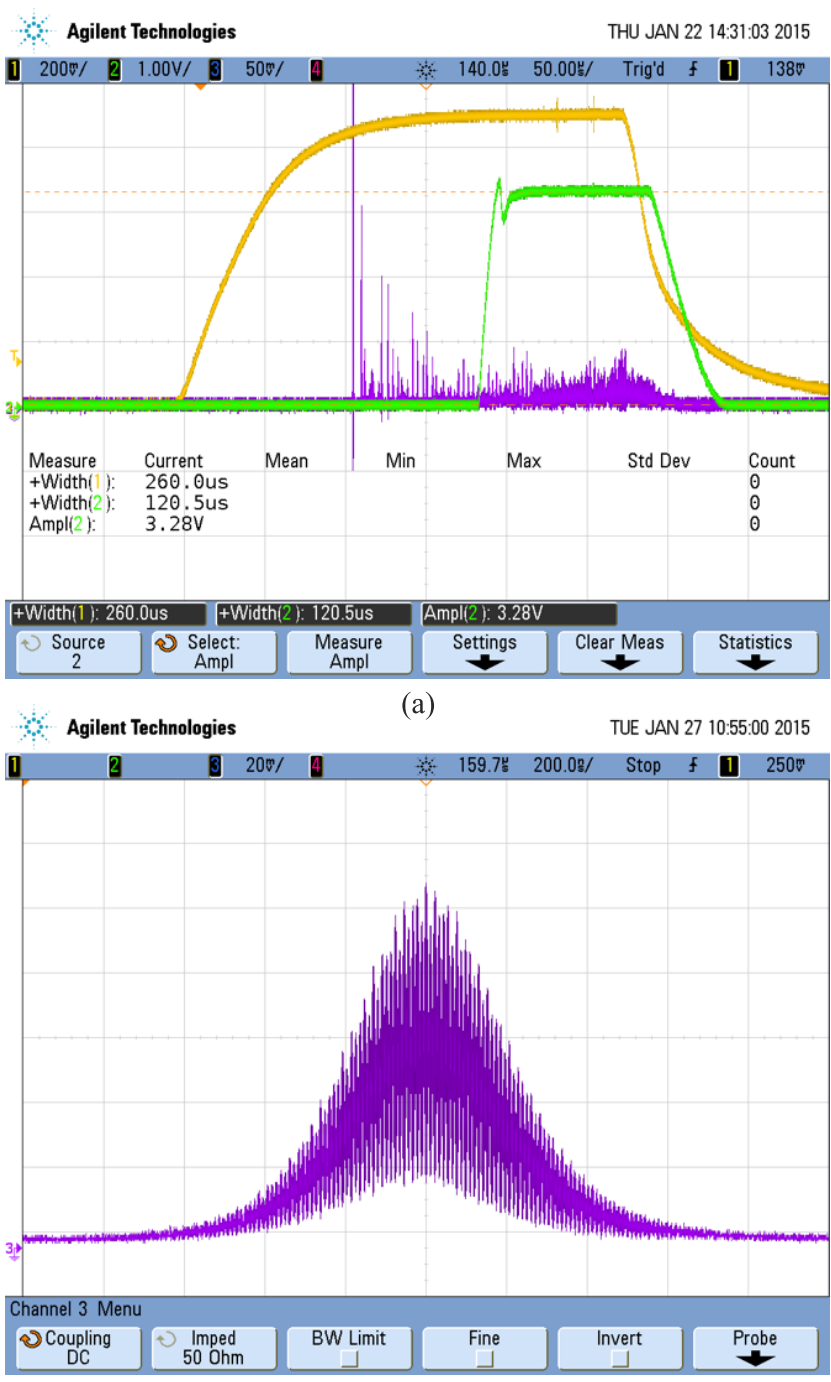

(b)

Fig. 6. Time trace of output pulse of the laser amplifier in relation to pump pulses (purple-laser; yellow - oscillator; green - amplifier).

\section{Conclusions}

We demonstrate a self-adaptive, nonreciprocal, open-loop Nd:YAG laser amplifier. In the experiments $228 \mathrm{~mJ}$ of output energy with a $17.8 \%$ slope efficiency was achieved. The NRTE did not enforce a single longitudinal mode in the cavity, hence each resonator mode forms own gain grating, what in turn creates a complex volumetric structure. The change of the wave plate retardation had no impact on the mode selection in the cavity. It only influenced on the threshold and efficiency of the laser. The optimization of the mode size and the overlap between interfering beams should increase the energy extraction efficiency. However, in the presence of high inhomogeneity of spatial gain distribution we managed to obtain near-diffraction-limited output with $M^{2}$ parameter less than 2.5 . In order to achieve an efficient, high-performance, high-energy and near-diffraction-limited

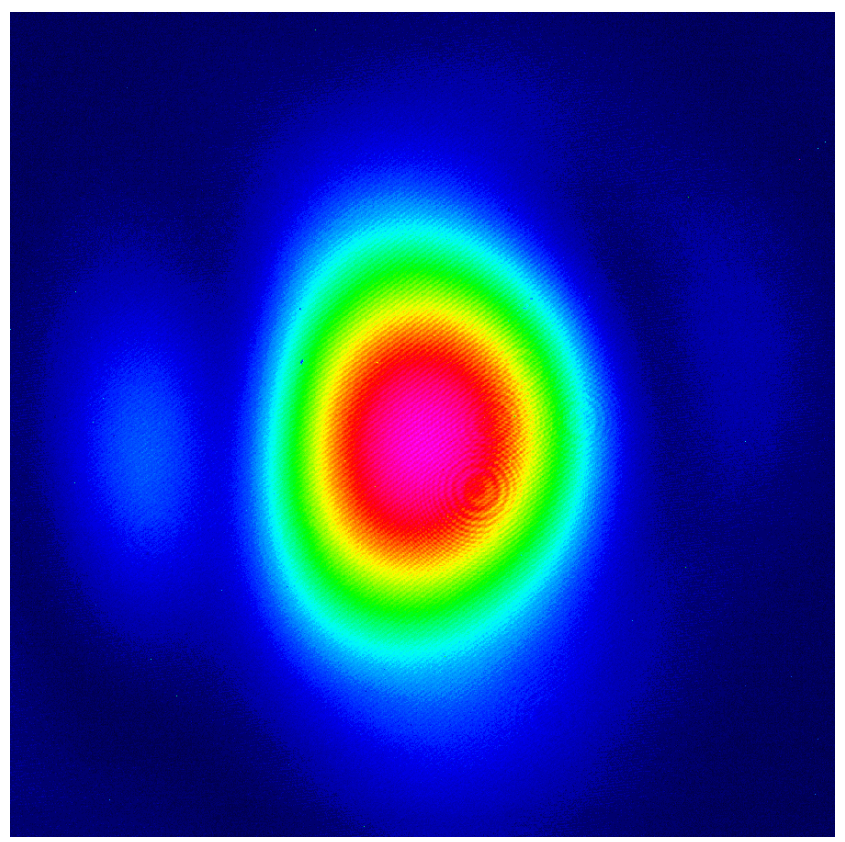

Fig. 7. Beam intensity spatial profile of a laser amplifier for $\theta=2.5 \mathrm{mrad}$.

quality, self-adaptive laser system, it is crucial to provide a single intra-cavity mode.

\section{Acknowledgements}

This work was supported by the Polish National Science Centre under the Project No. NCN2012/05/B/ST7/00088.

\section{References}

1. A.D. Farinas, E.K. Gustafson, and R.L. Byer, "Design and characterization of a $5.5-\mathrm{W}, \mathrm{cw}$, injection-locked, fibre-coupled, laser-diode-pumped Nd:YAG miniature-slab laser", Opt. Lett. 19, 114-116 (1994).

2. R.F. Teehan, J.C. Bienfang, and C.A. Denman, "Power scaling and frequency stabilization of an injection-locked Nd:YAG rod laser", Appl. Opt. 39, 3076-3084 (2000).

3. G.M. Thomas and M.J. Damzen, "Passively Q-switched $\mathrm{Nd}: \mathrm{YVO}_{4}$ laser with greater than $11 \mathrm{~W}$ average power", Opt. Exp. 19, 577-4582 (2011).

4. M. Kaskow, W. Zendzian, and J.K. Jabczynski, "Near-diffraction-limited, high peak power, electro-optically Q-switched, diode-side-pumped $\mathrm{Nd}: \mathrm{YVO}_{4}$ grazing-incidence oscillator", Opt. Laser Techn. 65, 50-55(2015).

5. O. L. Antipov, D.V. Chausov, A.S. Kuzhelev, V.A. Vorobev, and A.P. Zinoviev, "250-W average-power Nd:YAG Laser with self-adaptive cavity completed by dynamic refractive-index gratings", IEEE J. Quantum Electron. 37, 716-724 (2001).

6. M.J. Damzen, R.P.M. Green, and K.S. Syed, "Self-adaptive solid-state laser oscillator formed by dynamic gain-grating holograms", Opt. Lett. 20, 1704-1706 (1995).

7. P. Sillard, A. Brignon, and J.-P. Huignard, "Loop resonators with self-pumped phase-conjugate mirrors in solid-state saturable amplifiers", J. Opt. Soc. Am. B 14, 2049-2058 (1997). 
8. P. Sillard, A. Brignon, J.-P. Huignard, and J.-P. Pocholle, "Self-pumped phase-conjugate diode-pumped Nd:YAG loop resonator", Opt. Lett. 23, 1093-1095 (1998).

9. P. Sillard, A. Brignon, and J.P. Huignard, "Gain-grating analysis of a self-starting self-pumped phase-conjugate Nd:YAG loop resonator", IEEE J. Quantum Electron. 34, 465-472 (1998).

10. R. Soulard, A. Brignon, S. Raby, E. Durand, and R. Moncorge, "Diode-pumped Nd:YAG self-adaptive resonator with a high gain amplifier operating at $100 \mathrm{~Hz}$ ", Appl. Phys. B 106, 295-300 (2012).

11. T.T. Basiev, A.V. Gavrilov, M.N. Ershkov, S.N. Smetanin, A.V. Fedin, K.A. Bel'kov, A.S. Boreisho, and V.F. Lebedev, "Loop laser cavities with self-pumped phase-conjugate mirrors in low-gain active media for phase-locked multichannel laser systems", Quantum Electron. 41, 207-211 (2011).
12. A.P. Pogoda, T.B. Lebedeva, M.R. Yusopov, M.R. Liventsov, V.F. Lebedev, A.S. Boreysho, A.V. Gavrilov, S.N. Smetanin, and A.V. Fedin, "High power Nd:YAG laser with self-pumped phase-conjugate loop cavity and repetitive pulsed diode-matrix side-pumping", Proc. SPIE 8677, 86770Z (2013).

13. Z. Sun, Q. Li, Q. Cheng, M.J.H. Lei, and Y. Hui, "Efficient continuous diode-pumped $\mathrm{Nd}: \mathrm{YVO}_{4}$ slab laser with an opened-loop reciprocal dynamic holographic cavity", Zhongguo Jiguang/Chinese Journal of Lasers 41, 0702003 (2014).

14. W. Zendzian, J.K. Jabczynski, M. Kaskow, L. Gorajek, J. Kwiatkowski, and K. Kopczynski, " 250 mJ, self-adaptive, diode-side-pumped Nd:YAG slab laser", Opt. Lett. 37, 2598-2600 (2012).

15. M. Kaskow, W. Zendzian, J.K. Jabczynski, L. Gorajek, and M. Piasecki, "Passively Q-switched Nd:YAG laser with diffractive output resonator", Laser Phys. Lett. 11, 115813 (2014). 